\title{
An on-line NMF model for temporal pattern learning Theory with Application to Automatic Speech Recognition
}

\author{
Hugo Van hamme \\ University of Leuven, Department ESAT, Leuven, Belgium \\ hugo.vanhammedesat. kuleuven. be
}

\begin{abstract}
Convolutional non-negative matrix factorization (CNMF) can be used to discover recurring temporal (sequential) patterns in sequential vector non-negative data such as spectrograms or posteriorgrams. Drawbacks of this approach are the rigidity of the patterns and that it is intrinsically a batch method. However, in speech processing, like in many other applications, the patterns show a great deal of time warping variation and recognition should be online (possibly with some processing delay). Therefore, time-coded NMF (TC$\mathrm{NMF}$ ) is proposed as an alternative to CNMF to locate temporal patterns in time. TC-NMF is motivated by findings in neuroscience. The sequential data are first processed by a bank of filters such as leaky integrators with different time constants. The responses of these filters are modeled jointly by a constrained NMF. Algorithms for learning, decoding and locating patterns in time are proposed and verified with preliminary ASR experiments.
\end{abstract}

Keywords: non-negative matrix factorization, temporal patterns, integrate-andfire neurons, automatic speech recognition.

\section{$1 \quad$ Introduction}

Non-negative matrix factorization (NMF) [1] can be used to discover recurring patterns in non-negative data such as histograms, spectrograms or images. Multiple observations are projected to a vector space and stacked as the columns of a matrix $\mathbf{V}$ and approximated by the product $\mathbf{W H}$ of reduced rank, each with non-negative entries. The columns of $\mathbf{W}$ will contain the discovered one-dimensional patterns and $\mathbf{H}$ indicates their presence in the data. Because the non-negative patterns are combined with non-negative weights, the model can be thought of as a decomposition into parts. NMF and the related [2] PLSA [3] have been applied in many domains including image processing, speech enhancement, speech recognition, document clustering and term clustering.

Though this was not addressed in the original work in [1], the patterns to be discovered may show structure, such as adjacency of the pixels belong to a part. NMF disregards this structure by mapping the data to a one-dimensional vector and not imposing any a priori relation between the entries (the features) in this vector. Graph-

adfa, p. 1, 2011.

(c) Springer-Verlag Berlin Heidelberg 2011 
regularized NMF [4] is a generic extension that takes generic feature relations into account.

When the data have a sequential (two-dimensional) structure, such as spectrograms or, like the examples in this paper, time-varying neuron activations caused by spoken words, more specific formulations can be made. The frame stacking approach of stacking successive vectors [5] leads to suboptimal solutions because the repetition of features in successive data vectors or patterns is not exploited. Moreover, an inflation of the required number of model patterns corresponding to multiple time alignments is observed. Another solution is not to stack the data vectors of successive frames but rather to add them over a sliding window long enough to span the patterns, which has shown good results for modeling spoken digits in [6]. The disadvantage of this approach is that the event order within the patterns nor the order of occurrence of the patterns within a window are modeled. A more elegant solution is given by convolutional NMF (CNMF) [7,8], where the data are modeled as a sum of two-dimensional patterns, each convolved with an excitation function. While this model alleviates the objections to stacking, it suffers from the rigidity of the patterns. In speech processing, like in many other applications, the patterns show a great deal of variation. Successful models for word-sized patterns include some time warping mechanisms such as state alignments in hidden Markov models or dynamic time warping in exemplar-based speech recognition. For example, if the speaking rate is reduced, the convolutional patterns will tend to be too short and a good match with slow speech can only be obtained by averaging the patterns over all possible durations or by increasing the number of patterns. CNMF is also intrinsically a batch formulation requiring the complete input data to be known in order to decompose it. To recast it as an online method, block-wise approximations are required.

In this paper, an alternative NMF model, time coded NMF (TC-NMF), for pattern discovery in sequential data and subsequent decoding is proposed. Like CNMF, it is capable of modeling the sequential aspects of the patterns and it is capable of locating these patterns along the time axis. However, it does not model the data by rigid patterns, yet allows for a controllable temporal resolution. Instead, it feeds the nonnegative data into a bank of filters with different impulse responses and models their output jointly with an NMF. The relative output magnitudes of the different filters allow to locate patterns in time. Simply put, if the output of a sluggish filter is large, while that of a fast filter is small (large), the pattern that caused it must be far (close) to the current analysis time. This mechanism can be used to locate the patterns as a whole during decoding, but also to locate events within the patterns during pattern discovery.

The proposed model is plausible from the neuroscience perspective. Upstream neurons are tuned to fire on specific (acoustic) events. In early stages of auditory processing, the receptive field of a neuron corresponds to a specific time-frequency distribution. Further downstream, they respond to more complex temporal patterns. The firing rates of such neurons are the inputs to the TC-NMF. The neurons feed many integrate-and-fire (IaF) neurons [9] with exponentially fading action potentials [10]. Neurons with a long memory or slow decay accumulate spikes form the input neurons over a long period of time, a process known as temporal summation in neu- 
rophysiology. The firing rate of a IaF neuron will depend on the incoming spike frequency relative to its time constant. We assume IaF neurons come with a wide range of time constants. By this process, neurons further downstream that are connected to these IaF neurons will observe different inputs depending on how much time has elapsed since the occurrence of the (acoustic) event. They will effectively result in time-tuned filter behavior, which is proposed as a mechanism for time perception [13]. In the present work, the typical joint response on all IaF neurons is modeled by an NMF, a model that can be viewed as a neural network showing cognitively plausible properties such as lateral inhibition [11] and which can learn incrementally, i.e. without having to store all past training tokens [12]. An algorithm for computing the perceived time of the detected temporal patterns will be given.

\section{Model}

Consider non-negative data $v_{n t}(n=1 \ldots N)$ that vary with an index $t(t=1 \ldots T)$ which will be called time. In the experimental section, these will be posteriorgrams, but in principle, the proposed method applies equally well to any vector-valued sequential non-negative data such as magnitude spectrograms. The basic NMF model is based on the following generative model:

$$
v_{n t} \approx \sum_{r} w_{n r} h_{r t} \text { with } v_{n t} \geq 0, w_{n r} \geq 0, h_{r t} \geq 0
$$

At each time instant the observations are explained as a linear combination of $R$ onedimensional patterns $w_{n r}$. There is no temporal relation between the pattern weights $h_{r t}$. The one-dimensional pattern model looses the feature relations that exist in higher dimensional space. In some cases, it is adequate to extend (1) to a tensor decomposition [14], but this is not appropriate for spectrograms and most imaging data.

In convolutional NMF, the $r$-th pattern is 2-dimensional with a feature index $n$ and a time index $m\left(m=1 \ldots M_{r}\right)$ and is convolved with its time-dependent activation $h_{r t}$ :

$$
v_{n t} \approx \sum_{r} \sum_{m=0}^{M_{r}} w_{n m r} h_{r t}^{m \rightarrow}
$$

where $h_{r t}^{m \rightarrow}$ means that the sequence $h_{r t}$ is shifted to the right over $m$ samples of the index $t$, i.e. $h_{r t}^{m \rightarrow}=h_{r(t+m)}$ except that zeros are shifted in on the left $(t=1)$ and elements are lost on the right $(t=T)$. See [7] and [8]. The patterns have received a temporal model of duration $M_{r}$ and are accurately located in time by $h_{r t}$.

\subsection{Time-coded NMF}

To alleviate the disadvantages of CNMF mentioned in section 1, a model is proposed where the temporal data are first filtered by a bank of $K$ filters with decaying impulse response $f_{k}(t)(k=1 \ldots K)$ : 


$$
v_{n t}^{k}=\sum_{u=1}^{t} v_{n u} f_{k}(t-u)
$$

The decay functions could be the exponential family

$$
f_{k}(t)=e^{-\alpha_{k} t}
$$

a choice which is grounded in neuroscience (IaF neurons), which can easily be implemented as a bank of first order low-pass filters (leaky integrators) and which will be used in section 3 . The constants $\alpha_{k}$ are known, corresponding to the physical property of a specific neuron in the brain. An alternative choice could be a cascade of first order filters with equal time constant $f_{k}(t)=t^{k-1} e^{-\alpha t}$ which are reminiscent of Laguerre filters.

The TC-NMF model is derived by assuming $v_{n t}$ is composed of an additive combination of patterns located at times $\tau_{r t}$ having activation $h_{r t}$. Each pattern has an internal structure generating data valued at $p_{n s}^{r}$ at chosen times $q_{r s}$ relative to the end of the pattern:

$$
v_{n t}^{k} \approx g_{n t}^{k}+\sum_{r} \sum_{s=1}^{s_{r}} p_{n s}^{r} f_{k}\left(q_{r s}+\tau_{r t}\right) h_{r t}
$$

where $S_{r}$ controls the level of detail within the $r$-th pattern (much like $M_{r}$ in CNMF) and $g_{n t}^{k}$ will be explained in section 2.3. Approximating $f_{k}\left(q_{r s}+\tau_{r t}\right) \approx a_{s r k} f_{k}\left(\tau_{r t}\right)$ yields

$$
v_{n t}^{k} \approx g_{n t}^{k}+\sum_{r} w_{n r}^{k} f_{k}\left(\tau_{r t}\right) h_{r t} \triangleq d_{n t}^{k}
$$

where patterns are represented by $K$ vectors $w_{n r}^{k}$ irrespective of their internal structure or duration with:

$$
w_{n r}^{k}=\sum_{s=1}^{s_{r}} a_{s r k} p_{n s}^{r}
$$

For the first order filter bank (4), no approximation is involved and $a_{s r k}=e^{-\alpha_{k} q_{r s}}$

\subsection{Learning}

Learning is achieved by estimating the parameters $p_{n s}^{r}, \tau_{r t}$ and $h_{r t}$ by minimizing the sum over all $k$ of the Kullback-Leibler divergence (KLD) between the left hand side and the right hand side of (6). In principle, this can be done completely unsupervisedly, which has been successful on toy problems. On real speech data, utterance-level information was exploited to avoid local minima.

The update formulae are obtained by equating the partial derivative of the KLD w.r.t. the parameters to zero. Like with the original NMF, this leads to a fixed point multiplicative update for $p_{n s}^{r}$ : 


$$
p_{n s}^{r} \leftarrow p_{n s}^{r} \frac{\sum_{k} \sum_{t} \frac{v_{n t}^{k}}{d_{n t}^{k}} a_{s r k} f_{k}\left(\tau_{r t}\right) h_{r t}}{\sum_{k} \sum_{t} a_{s r k} h_{r t}}
$$

The update for $h_{r t}$ and $\tau_{r t}$ requires solving a $2 \times 2$ set of nonlinear equations. Form:

$$
\begin{aligned}
& \varphi_{r t}=\sum_{k} \sum_{n} \frac{v_{n t}^{k}}{d_{n t}^{k}} w_{n r}^{k} f_{k}\left(\tau_{r t}\right) h_{r t} \\
& \psi_{r t}=\sum_{k} \sum_{n} \frac{v_{n t}^{k}}{d_{n t}^{k}} w_{n r}^{k} f_{k}^{\prime}\left(\tau_{r t}\right) h_{r t}
\end{aligned}
$$

where prime denotes derivative. For the case of a leaky integrator filter bank, solve:

$$
\sum_{k}\left(1+\alpha_{k} \frac{\varphi_{r t}}{\psi_{r t}}\right)\left(\sum_{n} w_{n r}^{k}\right) e^{-\alpha_{k} \tau_{r t}}=0
$$

for $\tau_{r t}$ by a coarse search followed by a few Newton-Raphson updates. Then update

$$
h_{r t} \leftarrow \frac{\varphi_{r t}}{\sum_{k}\left(\sum_{n} w_{n r}^{k}\right) f_{k}\left(\tau_{r t}\right)}
$$

Updates (8), (11) and (12) are repeated until convergence. After each iteration $p_{n s}^{r}$ is normalized to sum to unity over $n$ and $s$ while $h_{r t}$ is normalized inversely to mitigate scale invariance. The KLD was always observed to decrease under these update rules but the convergence behavior remains to be studied from a theoretical point of view.

\subsection{Decoding}

Estimation of the activation of a pattern and its position is done with updates (11) and (12) with fixed $w_{n r}^{k}$ (or equivalently fixed $p_{n s}^{r}$ ). This will yield a different answer at every $t$ for which this problem is solved. Performing actual recognition requires an integration of these estimates into a global decision. A left-to-right decoding that does not involve dynamic programming is applied here and proceeds as follows. If $h_{r t}$ exceeds a threshold and its current $\tau_{r t}$ is positive (i.e. the pattern is observed completely for it ends earlier than the current time) and also places pattern $r$ after the last decoded pattern, pattern $r$ is accepted. To avoid that a pattern accepted earlier would be recognized again in the future, its future effect on $v_{n t}^{k}$ is predicted by the recursion (for leaky integrators):

$$
g_{n t}^{k} \leftarrow e^{-\alpha_{k}} g_{n t}^{k}+\sum_{\substack{\text { accepted } \\ r \text { at } t}} h_{r t} e^{-\alpha_{k} \tau_{r t}} w_{n r}^{k}
$$


where $g_{n t}^{k}$ is initialized to 0 . Adding $g_{n t}^{k}$ to (5) has the desired inhibitory effect and generates a signal model in which multiple occurrences of a pattern are modeled correctly.

\section{Application to speech recognition}

In this section, preliminary experiments on the adult speakers of the TIDIGITS corpus (strings of up to seven digits) are presented. Each of the 11 digits plus silence is considered as a pattern to be modeled. In order not to make the pattern estimation task trivial, the isolated digits are removed from the training set, leaving 6159 utterances from 112 speakers. For each utterance, a phone lattice is generated using an acoustic model and a bigram phonotactic model which are both trained on the 284 speakers of the Wall Street Journal corpus. The vertices of the lattice are labeled with frame number $t$ (= time at a $10 \mathrm{~ms}$ resolution) and the edges are labeled with one of 44 phone units and a posterior probability. The data $v_{n t}$ are composed of phone features $(1 \leq n \leq$ $44)$ and $44^{2}$ phone co-occurrence features $(45 \leq n \leq 1980=N)$. A phone feature is the posterior probability of phone $n$ at time $t$. The co-occurrence feature of phone pair $(A, B)$ is only non-zero of there is a vertex at time $t$ with an incoming edge with label $A$ and an outgoing edge with label $B$. The feature value is the product of their posterior probability. The bank of 17 first order filters uses damping coefficients $\alpha_{k}$ linearly spaced between 0.02 and 0.1 .

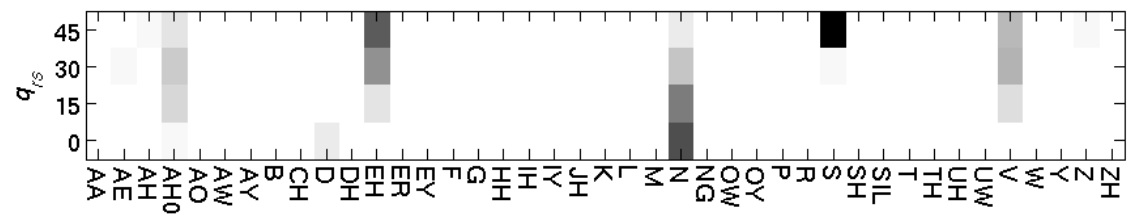

Fig. 1. Phone posterior part of the learned pattern model $p_{n s}^{r}$ for the digit "seven". Dark tones are large values. All phone labels were mapped to TIMIT symbols.

The training procedure is organized in several phases resulting in models with increased temporal detail. First $K=1$ and $\alpha_{1}=0$, which is unable to capture temporal detail in the patterns and $S_{r}=1$ (and $q_{r 1}=0$ ). At this point, utterance-level supervisory information is exploited by setting $h_{r t}=0$ for all not-occurring patterns (i.e. only word identity but not word order is used) in the estimation of $h_{r t}$ (one per utterance) and $p_{n 1}^{r}\left(\tau_{r t}\right.$ will not affect the cost function and is irrelevant). Then the full $K=17$ filters are applied and $h_{r t}$ and $\tau_{r t}$ are re-estimated to locate the patterns. Finally, the patterns are refined to $S_{r}=4$ with $q_{r s}=15(s-1)$ by re-estimation (initialization by duplication of $p_{n 1}^{r}$ ). Figure 1 shows an estimated phone posterior model in $p_{n s}^{r}$. The models correctly reflect the most likely phones from the beginning to the end of the words (e.g. ' $\mathrm{S}$ ' at the beginning $q_{r 1}=45$ ). On the test set with 6214 strings of at least two digits from 113 speakers (disjoint from the training set), 1.8\% substitutions and $1.9 \%$ insertions are observed, but also almost $13 \%$ deletions. This preliminary result was ob- 
tained without much optimization on parameters or feature sets. While the substitution rate is encouraging, the deletion problem may be due to the bottom-up decoding process that does not consider multiple hypotheses.

\section{Discussion and conclusion}

Like CNMF, TC-NMF allows to learn, recognize and locate temporal patterns in data. The solutions to the training and recognition problems were presented in this paper. At ach point in time, the pattern activation $h_{r t}$ can be estimated with their perceived times $\tau_{r t}$, using only the instantaneous data $v_{n t}^{k}$ which makes TC-NMF an on-line method. To conclude, some research directions are listed.

- Filter bank. The number of filters $K$ is not a critical parameter but should be greater than all $S_{r}$. The time constants $\alpha_{k}$ need to reflect the time scale of the patterns, but more research is required to suggest optimal values. Inappropriate constants or small $K$ lead to poor numerical conditioning of the $R$ matrices $a_{s r k}$ (rows $r$, columns $k$ ), which makes it impossible to infer the pattern model from data. The identifiability of patterns for other filter bank choices is not investigated.

- Representation of patterns. $S_{r}$ and $q_{r s}$ should be optimized for ASR. Parameter sharing among the pattern models could be considered.

- Robustness to timing and warping mismatch. Consider an observed pattern instance that is atypical in length (e.g. a slowly spoken digit). In CNMF and frame stacking, having a good match at the beginning of the pattern will imply that the observed features will not match at all towards the end. On spectrogram data for instance, a mismatch in the slope of a formant frequency trajectory will result in the different frequency channels (feature index $n$ ) holding the formant peak and hence extremely poor matches. In TC-NMF, a timing mismatch between model and data for pattern $r$ will result in a mismatch in the relative size of $f_{k}\left(\tau_{r t}\right) w_{n r}^{k}$ versus its contribution in $v_{n t}^{k}$, reflecting that close timing matches are better. However, it will not result in a mismatch along the feature dimension. Hence, a possible, today unconfirmed, advantage of TC-NMF could be robustness to timing or warping mismatch.

- Cascading TC-NMF. Observe that the input and the output to TC-NMF are alike: they are features with a time stamp. The output integrates inputs to larger, more complex units. Architectures that integrate several layers (e.g. spectra to phones to words) could be explored.

- Search. The proposed decoding mechanism maintains only one hypothesis, while traditional architectures for ASR examine a (cognitively implausible) huge set of hypotheses simultaneously. Augmenting the model with a search that does not take instantaneous irrevocable decisions is expected to be advantageous to accuracy and might facilitate the integration of a language model.

- Supervision. In the experiments, limited supervision information was used in the form of the identity of the keywords present in an utterance. The method is intrinsically unsupervised and could be applied as such. Like in many unsupervised NMF-based models, local minima of the cost could become problematic. 
Acknowledgement. This work is sponsored by IWT-SBO project 100049 (ALADIN) and KU Leuven research grant OT/09/028 (VASI).

\section{References}

1. Lee, D.D., Seung, H.S.: Learning the parts of objects by non-negative matrix factorization. Nature, vol. 401, no. 6755, pp. 788-791, 1999

2. Gaussier, E., Goutte, C.: Relation between PLSA and NMF and Implications. In: ACM Conference on Research and Development in Information Retrieval, SIGIR 2005, pp. 601602

3. Hofmann, T.: Probabilistic Latent Semantic Indexing, In: Proceedings of the TwentySecond Annual International SIGIR Conference on Research and Development in Information Retrieval (SIGIR-99), 1999

4. Cai, D., He, X., Han, T., Huang, T.: Graph regularized non-negative matrix factorization for data representation. IEEE Transactions on Pattern Analysis and Machine Intelligence, to appear, 2011.

5. Gemmeke, J.F., Virtanen, T., Hurmalainen, A.: Exemplar-based sparse representations for noise robust automatic speech recognition. IEEE Transactions on Audio, Speech and Language processing, vol. 19, no. 7, pp. 2067-2080, 2011

6. Stouten, V., Demuynck, K., Van hamme, H.: Discovering Phone Patterns in Spoken Utterances by Non-negative Matrix Factorisation. IEEE Signal Processing Letters, volume 15, pages 131-134, 2008.

7. Smaragdis, P.: Non-negative Matrix Factor Deconvolution; Extraction of Multiple Sound Sources from Monophonic Inputs. In C.G. Puntonet and A. Prieto (Eds.), IndependentComponent Analysis and Blind Signal Separation, 494-499, Springer Berlin / Heidelberg, 2004

8. O'Grady, P.D., Pearlmutter, B.A.: Discovering speech phones using convolutive nonnegative matrix factorisation with a sparseness constraint. Neurocomputing 72, 88-101, 2008

9. Gerstner, W., Kistler, M.W.: Spiking Neuron Models. Single Neurons, Populations, Plasticity. Cambridge University Press, 2002

10. Gluss, B.: A model for neuron firing with exponential decay of potential resulting in diffusion equations for probability density. Bulletin of Mathematical Biophysics, vol. 29, 233243, 1967.

11. Van hamme, H.. On the relation between perceptrons and non-negative matrix factorization. In SPARS 2011 Workshop : Signal Processing with Adaptive Sparse Structured Representations, Edinburgh, U.K., June 2011.

12. Driesen, J., Van hamme, H.: Modelling Vocabulary Acquisition, Adaptation and Generalization in Infants using Adaptive Bayesian PLSA. Neurocomputing, volume 74, No. 11, pages 1874--1882, 2011.

13. Heron, J., Aaen-Stockdale, C., Hotchkiss, J., Roach, N.W., McGraw, P.V., Whitaker, D.: Duration channels mediate human time perception. Proc. R. Soc. B, 2011. Doi: 10.1098/rspb.2011.1131

14. Cichocki A., Zdunek R., Phan A. H., Amari S.I.: Nonnegative matrix and Tensor Factorizations, Wiley, 2009 